\title{
Bruhat-Tits-Räume
}

\author{
Serge Lang
}

\begin{abstract}
Serge Lang wurde 1927 in Paris geboren, wo er auch seine ersten Schuljahre absolvierte. Die weitere Ausbildung erhielt er dann allerdings in den Vereinigten Staaten, wo er das California Institute of Technology (Caltech) und die University of Princeton besuchte. Hier erhielt er das Doktorat in Mathematik im Jahre 1951. Nach Aufenthalten am Institute for Advanced Study in Princeton und an der University of Chicago war er von 1955 bis 1970 Professor an der Columbia University in New York. Gastprofessuren in Princeton und Harvard folgten, und 1972 wurde er Professor an der Yale University. Seine Interessen sind weitgespannt, aber sein Hauptinteresse gehörte immer der Mathematik, besonders der Zahlentheorie. Bis anhin hat er 37 Bücher, mathematische und andere, und über 70 Forschungsartikel veröffentlicht.
\end{abstract}

Mit einem gewissen Recht könnte man sagen, der Ausgangspunkt des vorliegenden Beitrages von Serge Lang sei der folgende Satz: Auf einer Riemannschen Mannigfaltigkeit mit semi-negativer Krümmung besitzt eine kompakte Gruppe von Isometrien einen Fixpunkt. Aber dieser Satz, der von recht komplizierten Begriffen handelt, wird erst in den historischen Bemerkungen ganz am Schluss des Beitrages kurz zitiert (Abschnitt 4). Denn Serge Lang geht es hier um etwas anderes, nämlich um ein ganz elementares, erstaunlich allgemeines, aber dennoch weittragendes Resultat, das auf dem Umweg über den oben erwähnten abstrakten Satz entdeckt worden ist, den Fixpunktsatz von Bruhat-Tits (Theorem 1.2). Die Entdeckung dieses Fixpunktsatzes lag nicht auf der Hand, sie ergab sich erst nach einer längeren und gewundenen Entwicklung. Eine der dafür notwendigen Voraussetzungen war die Erkenntnis, dass zwischen dem komplizierten Begriff der semi-negativen Krümmung und dem einfachen SemiParallelogrammgesetz eine enge Beziehung besteht. Das Semi-Parallelogrammgesetz (Abschnitt 1), dessen Gültigkeit die definierende Eigenschaft der im Titel genannten Bruhat-Tits-Räume ist, verallgemeinert eine wohlbekannte Identität, die in der elementaren Vektoralgebra an einem Parallelogramm hergeleitet werden kann (siehe Figur 1). Serge Lang geht in den weiteren Teilen (Abschnitte 2 und 3) seines Beitrages auf ein explizites Beispiel eines Bruhat-Tits-Raumes ein. Diese beiden Abschnitte illustrieren auf attraktive Weise die Differentialrechnung in Vektorräumen, wie sie Mathematikstudierende in ihrem ersten Studienjahr erarbeiten. ust 
Das gewöhnliche Parallelogrammgesetz betrifft einen reellen Vektorraum versehen mit einem positiv definiten Skalarprodukt $(v, w) \mapsto v \cdot w$. In diesem Fall ist die Länge (Norm) eines Vektors $v$ durch

$$
|v|=\sqrt{v \cdot v}
$$

definiert, und eine kleine Rechnung liefert das Parallelogrammgesetz. Es besagt, dass für zwei Vektoren $v, w$ die Gleichung (siehe Figur 1)

$$
|v-w|^{2}+|v+w|^{2}=2|v|^{2}+2|w|^{2}
$$

erfüllt ist.

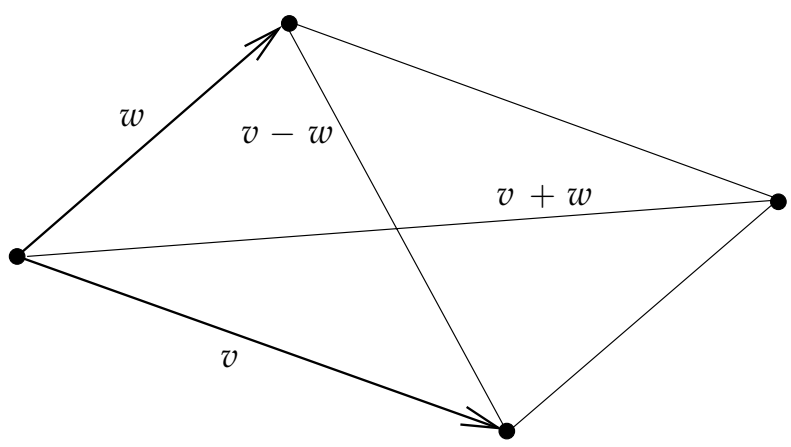

Fig. 1

Es ist bekannt, dass eine Vektorraum-Norm von einem positiv definiten Skalarprodukt herkommt, wenn die Norm das Parallelogrammgesetz erfüllt. Wir wollen uns hier aber nicht mit dieser Richtung der Gedanken beschäftigen. Sondern wir wollen einige neuere Entwicklungen darstellen, die zu durchsichtigen und überraschenden elementaren Resultaten geführt haben. Dabei kehren wir sozusagen die ursprüngliche, historische Abfolge der Entwicklungsschritte um, denn unsere Resultate stehen am Ende einer Kette von Gedankengängen, die speziellere und weit kompliziertere Situationen betreffen.

Der erste Abschnitt beschäftigt sich mit dem Semi-Parallelogrammgesetz. Im zweiten Abschnitt wird eine Metrik im Raum der reellen positiv definiten Matrizen definiert. Im dritten Abschnitt wird bewiesen, dass die übliche Exponentialabbildung auf symmetrischen Matrizen die Eigenschaft besitzt, Abstände zu vergrössern und dass der Raum der positiv definiten Matrizen das Semi-Parallelogrammgesetz erfüllt. Dies ist das klassische Beispiel. Im vierten Abschnitt unseres Beitrages gehen wir auf die Geschichte der Entwicklung dieses mathematischen Gebietes etwas näher ein; es zeigt sich dabei, dass diese Geschichte weder einfach, noch elementar ist. Aber so ist halt das Leben! Wir sollten uns aber durch die komplizierte Entwicklungsgeschichte nicht davon abhalten lassen, diejenigen Teile, die elementar zugänglich sind, einem grösseren Kreis bekannt $\mathrm{zu}$ machen.

Der Beitrag bringt Gegenstände zusammen, die im üblichen Mathematikstudium in verschiedenen elementaren Kursen berührt werden: Analysis, Lineare Algebra und Geometrie. Es ist Aufgabe dieser Kurse, eine breite Grundlage zu schaffen, und es bleibt deshalb 
in ihrem Rahmen kaum Zeit, sich mit speziellen Dingen eingehender zu befassen. Der vorliegende Beitrag schlägt eine mögliche Vertiefung von Begriffen und Resultaten vor, die in verschiedenen solchen Kursen vorkommen: die Ableitung als lineare Abbildung, die Exponentialreihe angewandt auf eine lineare Abbildung, positiv definite Matrizen, Länge von Kurven u.a. Eine Beschäftigung mit dem Inhalt des Beitrages sollte Studierenden der Mathematik in den unteren Semestern ohne weiteres möglich sein. Dies kann zum Beispiel in Gruppen von besonders Interessierten geschehen. In Yale habe ich auch mit Erfolg Studierende in einem speziellen Kolloquium über dieses Thema Vorträge halten lassen.

\section{Das Semi-Parallelogrammgesetz}

Im Folgenden betrachten wir einen metrischen Raum $X$ mit Abstandsfunktion $d$, so dass $d\left(x_{1}, x_{2}\right)$ den Abstand der Punkte $x_{1}, x_{2} \in X$ bezeichnet. Mit $\bar{B}_{r}\left(x_{1}\right)$ bezeichnen wir die abgeschlossene Kugel mit Radius $r$ und Zentrum $x_{1}$, also die Menge der Punkte $x \in X$ mit $d\left(x, x_{1}\right) \leq r$.

Natürlich ist in einem beliebigen metrischen Raum der Begriff eines Parallelogrammes nicht vorhanden. Um zum Semi-Parallelogrammgesetz zu gelangen, formulieren wir zuerst das ursprüngliche Parallelogrammgesetz (1) wie folgt um (siehe Figur 2):

$$
d\left(x_{1}, x_{2}\right)^{2}+4 d(x, z)^{2}=2 d\left(x, x_{1}\right)^{2}+2 d\left(x, x_{2}\right)^{2} .
$$

Dabei sind $x, x_{1}, x_{2}$ beliebig, und $z$ ist der Mittelpunkt der Strecke $x_{1} x_{2}$.

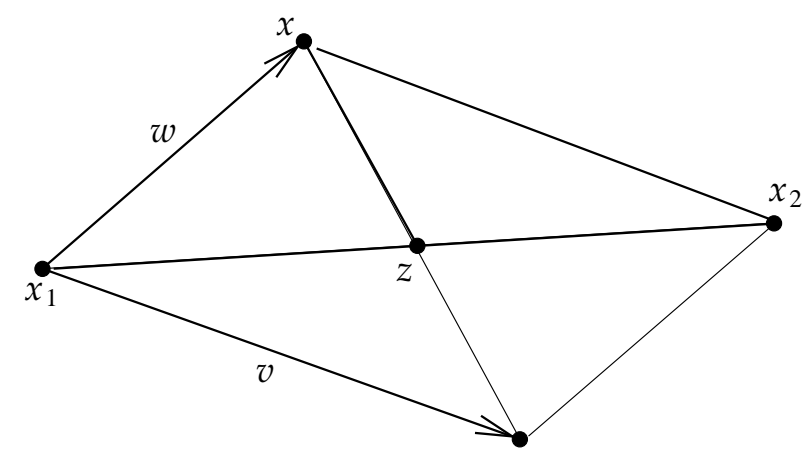

Fig. 2

Wir sagen nun, der metrische Raum $X$ erfülle das Semi-Parallelogrammgesetz, wenn für beliebige Punkte $x_{1}, x_{2} \in X$ ein Punkt $z$ existiert, so dass für alle $x \in X$ gilt

$$
d\left(x_{1}, x_{2}\right)^{2}+4 d(x, z)^{2} \leq 2 d\left(x, x_{1}\right)^{2}+2 d\left(x, x_{2}\right)^{2} .
$$

Aus dem Semi-Parallelogrammgesetz folgt

$$
d\left(z, x_{1}\right)=d\left(z, x_{2}\right)=\frac{1}{2} d\left(x_{1}, x_{2}\right) .
$$


Um dies einzusehen, setzt man im Semi-Parallelogrammgesetz zuerst $x=x_{1}$ und dann $x=x_{2}$. Man erhält so $2 d\left(x_{1}, z\right) \leq d\left(x_{1}, x_{2}\right)$ und $2 d\left(x_{2}, z\right) \leq d\left(x_{1}, x_{2}\right)$. Zusammen mit der Dreiecksungleichung

$$
d\left(x_{1}, x_{2}\right) \leq d\left(x_{1}, z\right)+d\left(z, x_{2}\right)
$$

ergibt sich dann die Gleichung (2).

Der Punkt $z$ ist durch $x_{1}$ und $x_{2}$ eindeutig bestimmt. Ist nämlich $z^{\prime}$ ein zweiter solcher Punkt, so setzen wir im Semi-Parallelogrammgesetz $x=z^{\prime}$ und erhalten sofort $z^{\prime}=z$. Aus diesem Grund nennen wir $z$ den Mittelpunkt zwischen $x_{1}$ und $x_{2}$.

Ein Bruhat-Tits-Raum ist definiert als ein vollständiger metrischer Raum, welcher das Semi-Parallelogrammgesetz erfüllt.

Theorem 1.1. (Serre) Es sei X ein Bruhat-Tits-Raum, und es sei S eine beschränkte Menge von $X$. Dann existiert eine eindeutig bestimmte Kugel von minimalem Radius $\bar{B}_{r}\left(x_{1}\right)$, die $S$ enthält: $S \subset \bar{B}_{r}\left(x_{1}\right)$.

Die Aussage von Theorem 1.1 ist wie folgt $\mathrm{zu}$ interpretieren: Ist $r$ das Infimum der reellen Zahlen $R \geq 0$, für die eine Kugel $\bar{B}_{R}\left(x^{\prime}\right)$ existiert, die $S$ enthält, so gibt es auch eine Kugel $\bar{B}_{r}\left(x_{1}\right)$, die $S$ enthält, und diese ist eindeutig bestimmt. Wir nennen $\bar{B}_{r}\left(x_{1}\right)$ die Umkugel und $x_{1}$ das Umkugelzentrum von $S$.

Beweis. Wir beweisen zuerst die Eindeutigkeit. Es seien $\bar{B}_{r}\left(x_{1}\right)$ und $\bar{B}_{r}\left(x_{2}\right)$ zwei Kugeln vom Radius $r$, die beide $S$ enthalten. Wir wählen $x \in S$. Dann gilt $d\left(x, x_{1}\right) \leq r$ und $d\left(x, x_{2}\right) \leq r$. Es sei $z$ der Mittelpunkt zwischen $x_{1}$ und $x_{2}$. Dann erhalten wir aus dem Semi-Parallelogrammgesetz die Ungleichung

$$
d\left(x_{1}, x_{2}\right)^{2} \leq 4 r^{2}-4 d(x, z)^{2}
$$

Nach Definition von $r$ als Infimum gibt es zu jedem $\epsilon>0$ einen Punkt $x \in S$ mit $d(x, z) \geq r-\epsilon$. Daraus folgt $d\left(x_{1}, x_{2}\right)=0$, also $x_{1}=x_{2}$.

Um die Existenz zu beweisen, betrachten wir eine Folge von Kugeln $\left\{\bar{B}_{r_{n}}\left(x_{n}\right)\right\}$, die alle $S$ enthalten und deren Radien $r_{n}$ gegen das Infimum $r$ streben. Wenn $\left\{x_{n}\right\}$ in $X$ eine Cauchy-Folge ist, so konvergiert diese im vollständigen Raum $X$ gegen einen Punkt; dieser ist der Mittelpunkt einer Kugel von minimalem Radius $r$, die $S$ enthält. Die Existenz einer solchen Kugel war nachzuweisen.

Es bleibt also zu beweisen, dass $\left\{x_{n}\right\}$ eine Cauchy-Folge ist. Dazu betrachten wir den Mittelpunkt $z_{m n}$ zwischen $x_{m}$ und $x_{n}$. Wegen der Minimalität von $r$ gibt es zu $\epsilon>0$ einen Punkt $x \in S$ mit

$$
d\left(x, z_{m n}\right)^{2} \leq r^{2}-\epsilon .
$$

Wir wenden nun das Semi-Parallelogrammgesetz an, wobei wir $z=z_{m n}$ setzen. Dann erhalten wir

$$
d\left(x_{m}, x_{n}\right)^{2} \leq 2 d\left(x, x_{m}\right)^{2}+2 d\left(x, x_{n}\right)^{2}-4 d\left(x, z_{m n}\right)^{2} \leq \epsilon(m, n)+4 \epsilon
$$


mit $\epsilon(m, n) \rightarrow 0$. Damit ist $\left\{x_{n}\right\}$ eine Cauchy-Folge, und der Beweis von Theorem 1.1 ist vollständig.

Es sei $X$ ein metrischer Raum. Unter einer Isometrie von $X$ verstehen wir eine bijektive Abbildung $g: X \rightarrow X$, so dass $g$ Abstände erhält, d.h. für alle $x_{1}, x_{2} \in X$ gilt

$$
d\left(g\left(x_{1}\right), g\left(x_{2}\right)\right)=d\left(x_{1}, x_{2}\right)
$$

Die Isometrien von $X$ bilden eine Gruppe. Denn die identische Abbildung ist eine Isometrie; sind $g_{1}$ und $g_{2}$ Isometrien, so ist auch deren Zusammensetzung $g_{1} \circ g_{2}$ eine Isometrie; und schliesslich ist die zu einer Isometrie inverse Abbildung wiederum eine Isometrie.

Es sei nun $G$ eine Gruppe von Isometrien des Raumes $X$, also eine Untergruppe der Gruppe aller Isometrien von $X$. Dem Gruppenelement $g \in G$ entspricht dann die Isometrie $x \mapsto g(x)$ von $X$. Man hat somit eine Operation der Gruppe $G$ auf $X$ durch Isometrien.

Für $x^{\prime} \in X$ bezeichnen wir mit $G x^{\prime}$ die Menge aller Elemente $g\left(x^{\prime}\right)$ mit $g \in G$. Wir nennen $G x^{\prime}$ die Bahnkurve (Orbit) von $x^{\prime}$ unter $G$. Es sei $S$ diese Bahnkurve. Für alle $g \in G$ und alle $x \in S$ folgt dann $g x \in S$. In der Tat gilt $x=g_{1} x^{\prime}$ für ein gewisses $g_{1} \in G$, und daraus ergibt sich $g\left(g_{1} x^{\prime}\right)=g\left(g_{1}\left(x^{\prime}\right)=\left(g \circ g_{1}\right)\left(x^{\prime}\right) \in S\right.$. Es ist sogar $g(S)=S$. Denn $g_{1} x^{\prime}$ kann geschrieben werden als $g_{1} x=g\left(g^{-1} g_{1}\right) x^{\prime}=g x, x=g^{-1} g\left(x^{\prime}\right)$. Wir sehen damit, dass $g \in G$ eine bijektive Abbildung von $S$ induziert.

Nach diesen Vorbereitungen können wir das folgende Theorem aussprechen.

Theorem 1.2. (Fixpunktsatz von Bruhat-Tits) Es sei X ein Bruhat-Tits-Raum, und es sei $G$ eine Gruppe von Isometrien von X. Es existiere für $G$ eine beschränkte Bahnkurve $S$ (dies ist zum Beispiel der Fall, wenn G kompakt ist). Dann besitzt G einen Fixpunkt, nämlich das Umkugelzentrum von $S$.

Beweis. Es sei $S$ die beschränkte Bahnkurve und $\bar{B}_{r}\left(x_{1}\right)$ die nach Theorem 1.1 eindeutig bestimmte Kugel mit minimalem Radius, die $S$ enthält. Da $g \in G$ eine Isometrie von $X$ induziert, ist $g\left(\bar{B}_{r}\left(x_{1}\right)\right)$ eine Kugel mit dem gleichen Radius $r$, also $g\left(\bar{B}_{r}\left(x_{1}\right)\right)=\bar{B}_{r}\left(x_{2}\right)$, $g\left(x_{1}\right)=x_{2}$. Wegen $g(S)=S$ enthält sie $S$. Aus der Eindeutigkeit folgt damit $g\left(x_{1}\right)=x_{1}$, so dass $x_{1}$ unter allen $g \in G$ invariant bleibt.

Theorem 1.1 ist ein sehr allgemeiner Fixpunktsatz; trotz dieser Allgemeinheit ist der Beweis erstaunlich einfach. Er ist so einfach, dass das Resultat selbst im Spezialfall eines euklidischen Raumes kaum einen einfacheren Beweis zulässt. Spezielle Resultate, die heute als Anwendungen von Theorem 1.1 angesehen werden können, waren lange bekannt. Aber erst in neuester Zeit ist schrittweise die einfache, "richtige" Formulierung des Resultates klar geworden, insbesondere wurde die zentrale Rolle, die das Semi-Parallelogrammgesetz in diesem Zusammenhang spielt, erst sehr spät erkannt. Wir kommen auf die interessante und komplizierte historische Entwicklung des Gebietes im letzten Abschnitt noch einmal zurück. 


\section{Der Raum der positiv definiten Matrizen}

Die Frage stellt sich, wie man Beispiele von Räumen finden kann, in denen zwar das Semi-Parallelogrammgesetz gilt, aber nicht das Parallelogrammgesetz. Es ist, grob gesprochen, die folgende geometrische Idee, die zu solchen Beispielen führt: Man beginnt mit einem Vektorraum, versehen mit einem positiv definiten Skalarprodukt; hier gilt das Parallelogrammgesetz. Dann versucht man, diesen Raum so zu "verbiegen" oder zu "krümmen", dass darin nur noch das Semi-Parallelogrammgesetz gilt. Was meint man mit "verbiegen" oder "krümmen"? Die genaue mathematische Definition des Begriffes "Krümmung", die hier eine Rolle spielt, ist leider zu kompliziert und würde auch mehr mathematische Grundlagen, erfordern, als dass man sie hier auf engem Raum anführen könnte. Hingegen lässt sich mit einfachen Mitteln ein Beispiel eines so "gekrümmten" Raumes angeben. Wir erzeugen diese "Krümmung" mit Hilfe der üblichen Exponentialabbildung, die wir auf den Vektorraum der symmetrischen Matrizen anwenden, und eines explizit gegebenen positiv definiten Skalarproduktes. Die Abschnitte 2 und 3 können als Beispiel für die elementare Differentialrechnung in Vektorräumen angesehen werden, als ein Beispiel allerdings, das von einem mathematisch komplizierteren und fortgeschritteneren Gegenstand handelt, als dies in einem Kurs über die Differentialrechnung in Vektorräumen üblich ist. Die Komplikation liegt aber einzig in der Art und Weise, wie wir das Material einordnen; die benötigten Techniken gehören durchaus zum Standardinhalt eines solchen Kurses.

$\mathrm{Zu}$ diesem Zweck betrachten wir den $n$-dimensionalen reellen Vektorraum $\mathbf{R}^{n}$, den Vektorraum der reellen $n \times n$-Matrizen $\mathbf{M a t}_{n}$ sowie den Vektorraum der symmetrischen reellen $n \times n$-Matrizen $\mathbf{S y m}_{n}$. Bezeichnet $\langle ， \quad\rangle$ das gewöhnliche Skalarprodukt in $\mathbf{R}^{n}$, so heisst eine Matrix $p \in \mathbf{S y m}_{n}$ positiv (oder positiv definit), wenn für alle $\xi \in \mathbf{R}^{n}, \xi \neq 0$ gilt

$$
\langle p \xi, \xi\rangle>0 \text {. }
$$

Zum Beispiel ist das Quadrat $v^{2}$ einer regulären Matrix $v \in \mathbf{S y m}_{n}$ immer positiv. Mit $\operatorname{Pos}_{n}$ bezeichnen wir die Menge der positiven symmetrischen reellen $n \times n$-Matrizen. Da reelle symmetrische Matrizen diagonalisierbar sind, sieht man, dass eine Matrix $v \in \mathbf{S y m}_{n}$ genau dann positiv ist, wenn ihre Eigenwerte alle positiv sind. Wir entnehmen daraus auch die Tatsache, dass eine Matrix in $\mathbf{P o s}_{n}$ immer regulär ist.

Als nächstes definieren wir die Exponentialabbildung von Matrizen. Für $v \in \mathbf{M a t}_{n}$ setzen wir

$$
\exp (v)=\sum_{n=0}^{\infty} \frac{v^{n}}{n !} .
$$

Die Reihe ist absolut konvergent. Um dies einzusehen, definiert man eine Norm auf Mat ${ }_{n}$ mit $|v w| \leq|v||w|$ für alle $v, w \in$ Mat $_{n}$. Der Beweis der Konvergenz von (3) verläuft dann analog zum Beweis der Konvergenz der Reihe für die reelle Exponentialfunktion. Wenn die Matrizen $v$ und $w$ kommutieren, $v w=w v$, so gilt $\exp (v+w)=\exp (v) \exp (w)$, so dass $\exp (v)$ invertierbar ist, die Inverse ist gegeben durch $\exp (-v)$. Damit kann exp als Abbildung

$$
\exp : \mathbf{M a t}_{n} \rightarrow \mathbf{G} \mathbf{L}_{n}
$$


angesehen werden, wo $\mathbf{G L}_{n}$ die Gruppe der regulären (invertierbaren) reellen $n \times n$ Matrizen bezeichnet. Für die symmetrische Matrix $v$ ist natürlich $\exp (v)$ wiederum symmetrisch; ausserdem folgt aus der letzten Bemerkung

$$
\exp (v)=\exp (v / 2) \exp (v / 2)=(\exp (v / 2))^{2}
$$

so dass $\exp (v)$ ein Quadrat und damit eine positive symmetrische Matrix ist. Damit liefert exp eine Abbildung

$$
\exp : \mathbf{S y m}_{n} \rightarrow \mathbf{P o s}_{n}
$$

die wir jetzt genauer untersuchen werden.

$\mathrm{Zu}$ exp lässt sich eine Umkehrabbildung definieren, die wir - in Analogie zum Fall der gewöhnlichen reellen Exponentialabbildung - Logarithmus nennen können. Um dies einzusehen, betrachten wir die positive symmetrische Matrix $p$. Da sich $p$ diagonalisieren lässt, existieren in $\mathbf{R}^{n}$ eine Basis $\left\{\xi_{1}, \xi_{2}, \ldots, \xi_{n}\right\}$ und Zahlen $\lambda_{1}, \lambda_{2}, \ldots, \lambda_{n}>0$ mit $p \xi_{i}=\lambda_{i} \xi_{i}$ für $i=1,2, \ldots, n$.

Man definiert dann $\log p=v$ als die Matrix der Abbildung, die in der Basis $\left\{\xi_{1}, \xi_{2}, \ldots, \xi_{n}\right\}$ durch die Matrix

$$
\left(\begin{array}{llll}
\log \lambda_{1} & & & \\
& \log \lambda_{2} & & \\
& & \ddots & \\
& & & \log \lambda_{n}
\end{array}\right)
$$

repräsentiert wird. In ganz analoger Weise kann auch die Quadratwurzel der Matrix $p$ definiert werden. Sie ist die Matrix der Abbildung, die in der Basis $\left\{\xi_{1}, \xi_{2}, \ldots, \xi_{n}\right\}$ durch die Matrix

$$
\left(\begin{array}{cccc}
\sqrt{\lambda}_{1} & & & \\
& \sqrt{\lambda}_{2} & & \\
& & \ddots & \\
& & & \sqrt{\lambda}_{n}
\end{array}\right)
$$

repräsentiert wird.

Im Folgenden werden wir zuerst im Vektorraum $\mathbf{S y m}_{n}$ ein (positiv definites) Skalarprodukt und damit eine Metrik einführen. Anschliessend werden wir in der Menge $\mathbf{P o s}_{n}$ eine Abstandsfunktion definieren, die diese Menge zu einem metrischen (topologischen) Raum macht. Schliesslich werden wir untersuchen, inwiefern die Abbildung $\exp : \mathbf{S y m}_{n} \rightarrow \mathbf{P o s}_{n}$ mit den eingeführten Metriken verträglich ist.

Das Skalarprodukt im Vektorraum $\mathbf{S y m}_{n}$ definieren wir mit Hilfe der Spur tr. Wir setzen für $v, w \in \mathbf{S y m}_{n}$

$$
\langle v, w\rangle_{\mathrm{tr}}=\langle v, w\rangle=\operatorname{tr}(v w)
$$

Diese Festsetzung definiert ein positiv definites Skalarprodukt: Die Zuordnung

$$
(v, w) \mapsto \operatorname{tr}(v w)
$$


ist offensichtlich bilinear; wegen $\operatorname{tr}(v w)=\operatorname{tr}(w v)$ ist sie symmetrisch, und schliesslich gilt für $v=\left(c_{i j}\right)=\left(c_{j i}\right)$ natürlich $\operatorname{tr}\left(v^{2}\right)=\sum c_{i j}^{2}$, so dass sie auch positiv definit ist.

Die zugehörige Norm ist durch

$$
|v|_{\operatorname{tr}}=|v|=\sqrt{\operatorname{tr}\left(v^{2}\right)}
$$

gegeben. Mit diesem Skalarprodukt wird $\mathbf{S y m}_{n}$ zu einem euklidischen Vektorraum (der Dimension $n(n+1) / 2)$ und damit in der üblichen Weise zu einem metrischen Raum. Der Abstand $d_{\mathbf{S y m}}(v, w)$ zwischen $v, w \in \mathbf{S y m}_{n}$ ist gegeben durch $|v-w|_{\mathrm{tr}}$.

$\mathbf{P o s}_{n}$ ist eine offene Untermenge von $\mathbf{S y m}_{n}$. Der Tangentialraum $T_{p}$ in einem Punkt $p \in \mathbf{P o s}_{n}$ ist eine Translationskopie von $\mathbf{S y m}_{n}$. Ohne komplizierte Terminologie zu verwenden, können wir ihn mit $\mathbf{S y m}_{n}$ identifizieren, genau gleich wie wir $\mathbf{R}^{N}$ mit dem Tangentialraum in irgend einem Punkt von $\mathbf{R}^{N}$ identifizieren. Wir definieren nun im Tangentialraum $T_{p}$ ein von $p \in \mathbf{P o s}_{n}$ abhängiges positives Skalarprodukt, indem wir

$$
\langle v, w\rangle_{p}=\operatorname{tr}\left(p^{-1} v p^{-1} w\right) .
$$

setzen. Die Tatsache, dass $\langle,\rangle_{p}$ positiv definit ist, ergibt sich wie folgt. Es ist

$$
\langle v, v\rangle_{p}=\operatorname{tr}\left(p^{-1} v p^{-1} v\right)=\operatorname{tr}\left(p^{-1 / 2} v p^{-1 / 2} p^{-1 / 2} v p^{-1 / 2}\right)=\operatorname{tr}\left(w^{2}\right)
$$

wobei wir $w=p^{-1 / 2} v p^{-1 / 2}$ gesetzt haben, und es gilt $\operatorname{tr}\left(w^{2}\right)>0$ für $v \neq 0$. Die zu diesem Skalarprodukt gehörige Norm ist durch

$$
|v|_{p}=\sqrt{\operatorname{tr}\left(\left(p^{-1} v\right)^{2}\right)}
$$

gegeben.

Ist $t \mapsto p(t)$ eine Kurve in $\mathbf{P o s}_{n}$, so ist die Ableitung der Bogenlänge gegeben durch

$$
\left(\frac{d s}{d t}\right)^{2}=\operatorname{tr}\left(\left(p(t)^{-1} p^{\prime}(t)\right)^{2}\right) .
$$

Damit ergibt sich die Länge $L(\alpha)$ einer stetig differenzierbaren Kurve

$$
\alpha:[a, b] \rightarrow \operatorname{Pos}_{n} ; t \mapsto \alpha(t)
$$

in $\mathbf{P o s}_{n}$ als Integral

$$
L(\alpha)=\int_{a}^{b}\left|\alpha^{\prime}(t)\right|_{\alpha(t)} d t .
$$

Diese Definition liefert in der üblichen Weise auch die Länge von stückweise stetig differenzierbaren Kurven in $\mathbf{P o s}_{n}$.

Um zu einer Metrik in $\mathbf{P o s}_{n}$ zu gelangen, definieren wir schliesslich den Abstand $d_{\text {Pos }}(p, q)$ zwischen zwei Punkten $p$ und $q$ von $\mathbf{P o s}_{n}$ als Infimum der Längen der stückweise stetig differenzierbaren Wege in $\mathbf{P o s}_{n}$, die $p$ und $q$ miteinander verbinden. 
Man kann ohne grosse Schwierigkeiten zeigen - wir wollen hier die notwendigen Rechnungen nicht explizit durchführen -, dass diese Festsetzung in der Menge $\mathbf{P o s}_{n}$ eine Abstandsfunktion definiert. Es ist nämlich in der Tat $d_{\mathbf{P o s}}($,$) positiv, symmetrisch$ und erfüllt die Dreiecksungleichung. Mit dieser Abstandsfunktion wird $\mathbf{P o s}_{n}$ zu einem metrischen Raum.

Der Vektorraum $\mathbf{S y m}_{n}$ besitzt eine Metrik, die durch die zum tr-Skalarprodukt gehörige Norm definiert ist. Er ist damit ein euklidischer Raum der Dimension $n(n+1) / 2$. Wir werden im Folgenden die bekannte Tatsache benützen, dass in einem euklidischen Raum der Abstand zweier Punkte durch die Länge der Verbindungsstrecke dieser beiden Punkte gegeben ist.

Die Exponentialabbildung

$$
\exp : \mathbf{S y m}_{n} \rightarrow \mathbf{P o s}_{n}
$$

liefert einen Diffeomorphismus zwischen den beiden Räumen. In Bezug auf die obige Metrik gilt das folgende Theorem, welches eines der Hauptresultate des vorliegenden Beitrages ist.

Theorem 2.1. Die Exponentialabbildung exp : $\mathbf{S y m}_{n} \rightarrow \mathbf{P o s}_{n}$ ist semi-dilatierend, d.h. es gilt für alle $v, w \in \mathbf{S y m}_{n}$

$$
d_{\mathbf{P o s}}(\exp (v), \exp (w)) \geq d_{\mathbf{S y m}}(v, w)=|v-w|_{\operatorname{tr}} .
$$

Die Exponentialabbildung ist also mit den Metriken in den beiden Räumen "semi-verträglich": die Abstände werden unter exp höchstens vergrössert.

Wir werden Theorem 2.1 im Abschnitt 3 beweisen. Hier wollen wir noch die folgenden Bemerkungen und Folgerungen anschliessen. Zuerst bemerken wir, dass es sich bei der Aussage von Theorem 2.1 um eine differentielle Eigenschaft der Abbildung exp handelt. Um das zu erklären, betrachten wir die Ableitung von exp:

$$
\exp ^{\prime}(v) w=\lim _{t \rightarrow 0} \frac{\exp (v+t w)-\exp (v)}{t}
$$

Dies liefert für jedes $v \in \mathbf{S y m}_{n}$ eine lineare Abbildung (der Tangentialräume)

$$
\exp ^{\prime}(v): \mathbf{S y m}_{n} \rightarrow \mathbf{S y m}_{n}
$$

Gilt nun - "differentiell" - für alle $w \in \mathbf{S y m}_{n}$

$$
\left|\exp ^{\prime}(v) w\right|_{\exp (v)} \geq|w|_{\mathrm{tr}},
$$

so folgt daraus die in Theorem 2.1 behauptete Eigenschaft der Abstandsfunktion. Dies ergibt sich direkt aus deren Definition als Infimum der Länge von Kurven, da ja die Länge einer Kurve mit Hilfe der Norm ||$_{p}$ im Tangentialraum definiert wird.

Aus Theorem 2.1 erhalten wir das folgende Korollar.

Korollar 2.2. Der metrische Raum Pos $_{n}$ ist vollständig. 
Beweis. Es sei $\left\{p_{k}\right\}$ eine Cauchy-Folge in $\mathbf{P o s}_{n}$ mit $p_{k}=\exp \left(v_{k}\right), v_{k} \in \mathbf{S y m}_{n}$. Aus Theorem 2.1 folgt, dass auch $\left\{v_{k}\right\}$ in $\mathbf{S y m}_{n}$ eine Cauchy-Folge ist. Letztere konvergiert deshalb gegen ein gewisses $v \in \mathbf{S y m}_{n}$. Wegen der Stetigkeit der Abbildung exp konvergiert dann $\left\{p_{k}\right\}=\left\{\exp \left(v_{k}\right)\right\}$ gegen $\exp (v)$. Damit ist die Vollständigkeit bewiesen.

Wir betrachten als nächstes eine Gerade durch den Ursprung von $\mathbf{S y m}_{n}$ in Richtung des Vektors $v, v \neq 0$, also die Kurve $t \mapsto t v, t \in \mathbf{R}$. Auf einer solchen Geraden gilt eine stärkere Aussage als Theorem 2.1, nämlich:

Theorem 2.3. Auf Geraden durch den Ursprung ist die Exponentialabbildung exp : $\mathbf{S y m}_{n} \rightarrow \mathbf{P o s}_{n}$ längentreu. Für $p \in \mathbf{P o s}_{n}$ mit $p=\exp v, v \in \mathbf{S y m}_{n}$ gilt

$$
d_{\mathbf{P o s}}(e, p)=d_{\mathbf{S y m}}(0, v)=|v|_{\text {tr }} .
$$

Beweis. Um die erste Behauptung zu beweisen, müssen wir $\left|\exp ^{\prime}(t v) v\right|_{\exp (t v)}$ berechnen. Nun gilt

$$
\frac{d}{d t} \exp (t v)=\exp ^{\prime}(t v) v=\frac{d}{d t} \sum \frac{t^{n} v^{n}}{n !}=\sum \frac{t^{n-1}}{(n-1) !} v^{n}=\exp (t v) v .
$$

Daraus folgt gemäss Definition

$$
\left|\exp ^{\prime}(t v) v\right|_{\exp (t v)}^{2}=\operatorname{tr}\left(\left((\exp (t v))^{-1}(\exp (t v) v)\right)^{2}\right)=\operatorname{tr}\left(v^{2}\right)=|v|_{\text {tr }}^{2} .
$$

Die Exponentialabbildung ist deshalb auf einer Geraden durch den Ursprung von $\mathbf{S y m}_{n}$ längentreu.

Was den zweiten Teil von Theorem 2.3 betrifft, also die globale Version der Aussage, so ergibt sich dieser aus Theorem 2.1. Es sei $p=\exp v$. Es ist $|v|_{\text {tr }}$ die Länge der Verbindungsstrecke von 0 und $v$. Um $d(e, p)=|v|_{\text {tr }}$ zu zeigen, betrachten wir eine stückweise stetig differenzierbare Kurve $\alpha$ in $\operatorname{Pos}_{n}$, die $e$ mit $p$ verbindet; sie habe die Länge L. Da exp invertierbar ist, ist die Kurve $\alpha$ Bild einer Kurve in $\mathbf{S y m}_{n}$, die 0 mit $v$ verbindet. Nach Theorem 2.1 gilt dann

$$
|v|_{\text {tr }}=d_{\text {Sym }}(0, v) \leq d_{\mathbf{P o s}}(e, p) \leq L .
$$

Es folgt, dass das Bild unter exp der Verbindungstrecke zwischen 0 und $v$ eine Kurve kürzester Länge zwischen $e$ und $p$ ist. Deren Länge ist definitionsgemäss $d_{\text {Pos }}(e, p)$; nach obigem ist sie gerade $|v|_{\text {tr }}$. Damit ist auch die globale Version der Aussage bewiesen.

Mit Hilfe dieses Resultates können wir beweisen, dass in $\mathbf{P o s}_{n}$ das Semi-Parallelogrammgesetz "im Ursprung" gilt, genauer:

Theorem 2.4. Es sei $0 \neq v_{1} \in \mathbf{S y m}_{n}$ und $v_{2}=-v_{1}$. Wir setzen $x_{1}=\exp \left(v_{1}\right), x_{2}=$ $\exp \left(v_{2}\right)$ und $z=\exp (0)=e$. Dann folgt für $v \in \mathbf{S y m}_{n}$ und $x=\exp (v)$

$$
d_{\text {Pos }}\left(x_{1}, x_{2}\right)^{2}+4 d_{\mathbf{P o s}}(x, e)^{2} \leq 2 d_{\mathbf{P o s}}\left(x, x_{1}\right)^{2}+2 d_{\mathbf{P o s}}\left(x, x_{2}\right)^{2} .
$$


Beweis. Im Vektorraum $\mathbf{S y m}_{n}$ gilt das Parallelogrammgesetz

$$
d_{\mathbf{S y m}}\left(v_{1}, v_{2}\right)^{2}+4 d_{\mathbf{S y m}}(v, 0)^{2}=2 d_{\mathbf{S y m}}\left(v, v_{1}\right)^{2}+2 d_{\mathbf{S y m}}\left(v, v_{2}\right)^{2} .
$$

Unter der Exponentialabbildung exp werden nach Theorem 2.3 die Abstände auf der linken Seite erhalten, während die Abstände auf der rechten Seite nach Theorem 2.1 gleich bleiben oder vergrössert werden. Damit erhalten wir genau die Aussage des SemiParallelogrammgesetz in $\mathbf{P o s}_{n}$ für diesen Spezialfall.

Um zu zeigen, dass $\mathbf{P o s}_{n}$ ein Bruhat-Tits-Raum ist, müssen wir noch nachweisen, dass das Semi-Parallelgesetz nicht nur "im Ursprung" sondern allgemein gültig ist. Zu diesem Zweck benützen wir die Tatsache, dass die allgemeine lineare Gruppe $\mathrm{GL}_{n}$ auf $\mathbf{P o s}_{n}$ operiert. Dies wird uns erlauben, die Aussage von Theorem 2.4 auf beliebige Punkte zu übertragen.

Die Gruppe $G=\mathrm{GL}_{n}$ der invertierbaren $n \times n$-Matrizen über $\mathbf{R}$ operiert auf $\mathbf{P o s}_{n}$ wie folgt. Für $g \in G$ definieren wir

$$
[g] p=g p^{t} g, \quad p \in \mathbf{P o s}_{n}
$$

Dabei bezeichnet ${ }^{t} g$ wie üblich die zu $g$ transponierte Matrix. Die Matrix $g p^{t} g$ ist positiv. Denn für alle $0 \neq \xi \in \mathbf{R}^{n}$ gilt ${ }_{g \xi} g \neq 0$ und

$$
\langle[g] p \xi, \xi\rangle=\left\langle g p^{t} g \xi, \xi\right\rangle=\left\langle p^{t} g \xi,{ }^{t} g \xi\right\rangle>0 .
$$

Damit ist $[g]$ eine Abbildung

$$
[g]: \mathbf{P o s}_{n} \rightarrow \mathbf{P o s}_{n} .
$$

Da - wie man leicht zeigt - für $g_{1}, g_{2} \in G$

$$
\left[g_{1}^{-1}\right]=\left[g_{1}\right]^{-1}, \text { und }\left[g_{1} g_{2}\right]=\left[g_{1}\right]\left[g_{2}\right]
$$

gilt, haben wir eine Operation der Gruppe $G$ auf $\mathbf{P o s}_{n}$ vor uns.

Wir stellen zuerst fest, dass diese Operation transitiv ist, d.h. dass zu beliebigen $p_{1}, p_{2} \in$ $\operatorname{Pos}_{n}$ ein $g \in G$ existiert mit $[g] p_{1}=p_{2}$. Ist nämlich $p=q^{2}$ mit $q$ positiv, so folgt $p=[q] e$. Mit $p_{1}=\left[q_{1}\right] e$ und $p_{2}=\left[q_{2}\right] e$ erhalten wir aber sofort $p_{2}=\left[q_{2} q_{1}^{-1}\right] p_{1}$. Damit ist $g=q_{2} q_{1}^{-1} \in G$ das gesuchte Element.

Theorem 2.5. Für $g \in G$ ist die Abbildung $p \mapsto[g] p$ von $\mathbf{P o s}_{n}$ eine Isometrie.

Beweis. Wir bemerken zuerst, dass $[g]$ auch als Abbildung auf dem ganzen Vektorraum Sym betrachtet werden kann, und dass diese Abbildung eine lineare Funktion dieser Matrizen ist. Die Ableitung ist deshalb für $w \in \mathbf{S y m}$ durch

$$
[g]^{\prime}(p) w=g w^{t} g
$$


gegeben. Wir verifizieren jetzt, dass $[g]$ das Skalarprodukt erhält. Wir haben:

$$
\begin{aligned}
\left|[g]^{\prime}(p) w\right|_{[g] p}^{2} & =\operatorname{tr}\left(\left((g g p)^{-1} g w^{t} g\right)^{2}\right) \\
& =\operatorname{tr}\left(\left(\left(g p p^{t} g\right)^{-1}\left(g w^{t} g\right)\right)^{2}\right) \\
& =\operatorname{tr}\left({ }^{t^{-1}} p^{-1} g^{-1} g w^{t} g^{t} g^{-1} p^{-1} g^{-1} g w^{t} g\right) \\
& =\operatorname{tr}\left({ }^{t}{ }^{-1} p^{-1} w p^{-1} w^{t} g\right) \\
& =\operatorname{tr}\left(p^{-1} w p^{-1} w\right) \\
& =|w|_{p}^{2} .
\end{aligned}
$$

Dies beweist das Theorem.

Der Beweis des folgenden Theorems verläuft ähnlich wie der Beweis von Theorem 2.5. Wir überlassen ihn deshalb dem Leser.

Theorem 2.6. Die Abbildung $p \mapsto p^{-1}$ von Pos $_{n}$ ist eine Isometrie.

Die beiden letzten Resultate erlauben es, eine explizite Formel für den Abstand zweier Punkte in $\mathbf{P o s}_{n}$ herzuleiten (siehe Theorem 2.7). Ausserdem ermöglichen sie zu zeigen, dass das Semi-Parallelogrammgesetz allgemein, und nicht nur im Ursprung, gültig ist (siehe Theorem 2.8).

Theorem 2.7. Für $p, q \in \mathbf{P o s}_{n}$ seien $a_{1}, a_{2}, \ldots, a_{n}$ die Nullstellen des Polynoms $\operatorname{det}(t p-q)$. Dann gilt

$$
d_{\mathbf{P o s}}(p, q)=\sqrt{\sum\left(\log a_{i}\right)^{2}}
$$

Beweis. Wir betrachten zuerst den Spezialfall mit $p=e$ und $q=d$, wo $d$ die Diagonalmatrix mit Diagonalelementen $a_{1}, a_{2}, \ldots, a_{n}$ ist. Es sei $v$ die Diagonalmatrix mit Diagonalelementen $\log a_{1}, \log a_{2}, \ldots, \log a_{n}$. Dann gilt $q=\exp (v)$. Gemäss Theorem 2.3 haben wir dann

$$
d_{\mathbf{P o s}}(e, d)=d_{\mathbf{S y m}}(0, v)=|v|_{\text {tr }}=\sqrt{\sum\left(\log a_{i}\right)^{2}} .
$$

Dies beweist die Aussage des Satzes im Spezialfall. Der allgemeine Fall wird nun auf diesen Spezialfall zurückgeführt. Zuerst finden wir $g \in G$ mit $[g] p=e$ und $[g] q=d$, wo $d$ eine Diagonalmatrix ist. Wegen der Transitivität der Operation existiert $h \in G$ mit $[h] p=e$. Es sei $q^{\prime}=[h] q$. Zu $q^{\prime}$ existiert eine orthonormale Basis in $\mathbf{R}^{n}$, welche $q^{\prime}$ diagonalisiert. Es existieren also eine Diagonalmatrix $d$ und eine orthogonale Matrix $k$ mit $d=k q^{\prime} k^{-1}=k q^{\prime}{ }^{t} k=[k] q^{\prime}$, denn für eine orthogonale Matrix $k$ ist $k^{-1}={ }^{t} k$. Wegen $e=k k^{-1}=k^{t} k$ folgt für $g=k h$, sofort $[g] p=[k] e=e$ und $[g] q=[k] q^{\prime}=d$. Umgekehrt gilt $p=g^{-1 t} g^{-1}$ und $q=g^{-1} d^{t_{g}-1}$, so dass folgt

$$
\operatorname{det}(t p-q)=\operatorname{det}\left(t g^{-1} g^{-1}-g^{-1} d^{t} g^{-1}\right)=(\operatorname{det} g)^{-2} \operatorname{det}(t e-d) .
$$


Die Nullstellen von $\operatorname{det}(t p-q)$ stimmen also mit den Nullstellen von $\operatorname{det}(t e-d)$ überein, und diese letzteren sind gerade die Diagonalelemente von $d$. Mit Theorem 2.5 folgt ausserdem $d_{\mathbf{P o s}}(e, d)=d_{\mathbf{P o s}}(p, q)$. Damit ist der Beweis von Theorem 2.7 vollständig.

Theorem 2.8. Der Raum Pos $_{n}$ ist ein Bruhat-Tits-Raum, d.h. er erfüllt das Semi-Parallelogrammgesetz.

Beweis Wir führen hier den allgemeinen Fall mit Hilfe der Operation von $G$ auf den in Theorem 2.4 erledigten Spezialfall zurück. Da $G$ durch Isometrien operiert, genügt es zu zeigen, dass zu $x_{1}, x_{2} \in \mathbf{P o s}_{n}$ ein $g \in G$ existiert mit $[g] x_{1}=\exp \left(v_{1}\right)$ und $[g] x_{2}=\exp \left(-v_{1}\right)$ für ein gewisses $v_{1} \in \mathbf{S y m}_{n}$. Wegen der Transitivität der Operation von $G$ auf $\operatorname{Pos}_{n}$, existiert $h \in G$ mit $[h] x_{2}=e$; es sei $[h] x_{1}=p$. Wir setzen dann $k=p^{-1 / 4}$ mit $k \in \mathbf{P o s}_{n}$; natürlich ist $k$ symmetrisch. Es folgt $[k] p=p^{1 / 2}$ und $[k] e=p^{-1 / 2}$. Setzen wir also $g=k h$, so gilt $[g] x_{1}=p^{1 / 2}$ und $[g] x_{2}=p^{-1 / 2}$. Schliesslich sei $p^{1 / 2}=\exp \left(v_{1}\right)$ für $v_{1} \in \mathbf{S y m}_{n}$. Dann ist $v_{1}$ das gesuchte Element. Damit ist Theorem 2.8 vollständig bewiesen.

\section{Die Exponentialabbildung ist semi-dilatierend}

In diesem Abschnitt geben wir einen Beweis von Theorem 2.1. Wir halten dabei $n$ fest und schreiben deshalb Mat für $\mathbf{M a t}_{n}$ und $\mathbf{S y m}$ für $\mathbf{S y m}_{n}$.

Für $v, w \in$ Mat definieren wir

$$
F_{v}(w)=\exp (-v / 2) \cdot \exp ^{\prime}(v) w \cdot \exp (-v / 2)
$$

Man beachte

$$
\exp ^{\prime}(v) w=\left.\frac{d}{d t} \exp (v+t w)\right|_{t=0} .
$$

Man erhält dann direkt aus der Definition

$$
\exp ^{\prime}(v) w=\sum_{n=0}^{\infty} \frac{1}{n !} \sum_{r+s=n-1} v^{r} w v^{s}
$$

Da Potenzen eines Elementes kommutieren, sehen wir ferner, dass $\exp (-v / 2)$ mit den Potenzen $v^{r}$ und $v^{s}$ kommutiert.

Lemma 3.1. Die linearen Abbildungen $F_{v}$ und $\exp ^{\prime}(v)$ sind selbstadjungiert bezüglich des tr-Skalarproduktes in Mat. Für $v \in \mathbf{S y m}$ bilden $F_{v}$ und $\exp (v)$ den Vektorraum $\mathbf{S y m}$ in sich $a b$.

Beweis. Eine Rechnung liefert für $u, v, w \in$ Mat

$$
\begin{aligned}
\operatorname{tr}\left(F_{v}(w) u\right) & =\sum_{n=0}^{\infty} \frac{1}{n !} \sum_{r+s=n-1} \operatorname{tr}\left(\exp (-v / 2) v^{r} w v^{s} \exp (-v / 2) u\right) \\
& =\sum_{n=0}^{\infty} \frac{1}{n !} \sum_{r+s=n-1} \operatorname{tr}\left(w v^{s} \exp (-v / 2) u \exp (-v / 2) v^{r}\right) \\
& =\operatorname{tr}\left(w F_{v}(u)\right)
\end{aligned}
$$


da $\exp (-v / 2)$ mit $v^{r}$ und $v^{s}$ vertauscht. Damit ist gezeigt, dass die Abbildung $F_{v}$ bezüglich des tr-Skalarproduktes selbstadjungiert ist.

Für $v \in \mathbf{S y m}$ folgt direkt aus der Definition, dass die Abbildung $F_{v}$ den Vektorraum Sym in sich abbildet.

Die Aussagen über $\exp (v)$ werden ganz analog bewiesen; wir verzichten deshalb darauf, den Beweis auszuführen.

Für $v \in$ Mat definieren wir die Linksmultiplikation $L_{v}:$ Mat $\rightarrow$ Mat durch $L_{v}(w)=v w$, $w \in$ Mat, und analog die Rechtsmultiplikation $R_{v}:$ Mat $\rightarrow$ Mat durch $R_{v}(w)=w v$. Im Folgenden wird die Abbildung $D_{v}=L_{v}-R_{v}$ eine grosse Rolle spielen. Für $w \in$ Mat gilt

$$
D_{v}(w)=v w-w v=[v, w] .
$$

Lemma 3.2. Für $v \in \mathbf{S y m}$ ist die lineare Selbstabbildung $D_{v}^{2}$ von $\mathbf{S y m}$ selbstadjungiert bezüglich des tr-Skalarproduktes.

Beweis. Es gilt

$$
\begin{aligned}
D_{v}(w) & =v w-w v \\
D_{v}^{2}(w) & =v^{2} w-2 v w v+w v^{2} \\
D_{v}^{2}(w) u & =v^{2} w u-2 v w v u+w v^{2} u \\
w D_{v}^{2} u & =w v^{2} u-2 w v u v+w u v^{2}
\end{aligned}
$$

Die Anwendung von tr auf die letzten zwei Gleichungen führt dann wegen der Eigenschaft $\operatorname{tr}(x y)=\operatorname{tr}(y x)$ sofort zum gewünschten Resultat.

Ein symmetrischer Operator $B$ auf einem euklidischen Vektorraum $V$, also eine selbstadjungierte Selbstabbildung von $V$, heisst semi-positiv, wenn für alle $w \in V$ gilt

$$
\langle B w, w\rangle \geq 0 .
$$

Sind $B_{1}$ und $B_{2}$ zwei symmetrischen Operatoren auf $V$, so setzen wir $B_{1} \geq B_{2}$, falls gilt $B_{1}-B_{2} \geq 0$. Wir bezeichnen weiter mit $I=I_{V}: V \rightarrow V$ die identische Abbildung von $V$.

Im schrittweisen Beweis von Theorem 2.1 werden wir die folgenden zwei grundlegenden Eigenschaften brauchen.

Spektraleigenschaft. Es sei $M$ ein symmetrischer Operator auf dem euklidischen Vektorraum $V$, und es sei $b \geq 0, b \in \mathbf{R}$. Es sei ferner $f_{0}(t)$ eine konvergente Potenzreihe mit $f_{0}(t) \geq b$ für alle $t$ in einem Intervall, das die Eigenwerte von $M$ enthält. Dann gilt $f_{0}(M) \geq b I$.

Der Beweis folgt direkt durch Diagonalisierung von $M$.

Die Schwarzsche Eigenschaft. Für alle $v, w \in \mathbf{S y m}$ gilt

$$
\operatorname{tr}\left((v w)^{2}\right) \leq \operatorname{tr}\left(v^{2} w^{2}\right)
$$


Beweis. Die symmetrischen Matrizen $v$ und $w$ können simultan diagonalisiert werden, wenn eine davon positiv definit ist. In diesem Fall ist die Aussage nichts anderes als die übliche Schwarzsche Ungleichung. Sind beide Matrizen singulär, so betrachten wir die Matrix $v+\epsilon e$ mit $\epsilon>0$ und $e$ die Einheitsmatrix. Für alle genügend kleinen $\epsilon \neq 0$ ist die Matrix $v+\epsilon e$ regulär. Man benützt dann das Resultat für den regulären Fall und schliesst einen Grenzübergang $\epsilon \rightarrow 0$ an. Damit ist der Beweis der Schwarzschen Eigenschaft vollständig.

Wir betrachten als nächstes die Potenzreihe

$$
f(t)=\sum_{k=0}^{\infty} \frac{(t / 2)^{2 k}}{(2 k+1) !}=\frac{\sinh (t / 2)}{t / 2}=\frac{\exp (t / 2)-\exp (-t / 2)}{t}
$$

Wir bemerken, dass die Abbildungen $L_{v}$ und $R_{v}$ kommutieren, so dass gilt

$$
\left(\exp D_{v} / 2\right)=\left(\exp L_{v} / 2\right) \cdot \exp \left(-R_{v} / 2\right) .
$$

Wir untersuchen nun $f\left(D_{v}\right)$. Aus Lemma 3.2 folgt, dass für $v \in \mathbf{S y m}$ jede gerade Potenz von $D_{v}$ bezüglich des tr-Skalarproduktes in Sym ein selbstadjungierter Operator ist. Da in der Potenzreihe $f(t)$ nur gerade Potenzen auftreten, ist folglich für $v \in \mathbf{S y m}$ auch $f\left(D_{v}\right)$ ein selbstadjungierter Operator auf Sym.

Lemma 3.3. Für jedes $v \in$ Mat gilt $D_{v} F_{v}=D_{v} f\left(D_{v}\right)$.

Beweis. Es sei $t \mapsto x(t)$ eine differenzierbare Kurve in Mat. Wir betrachten

$$
x(\exp x)=(\exp x) x
$$

und differenzieren. Wir erhalten

$$
x^{\prime}(\exp x)+x(\exp x)^{\prime}=(\exp x)^{\prime} x+(\exp x) x^{\prime} .
$$

Daraus folgt

$$
x^{\prime}(\exp x)-(\exp x) x^{\prime}=(\exp x)^{\prime} x-x(\exp x)^{\prime} .
$$

Multiplikation von links und rechts mit $\exp (-x / 2)$ liefert, zusammen mit der Tatsache, dass $x$ mit $\exp (-x / 2)$ kommutiert,

$$
\begin{aligned}
& \exp (-x / 2) x^{\prime} \exp (x / 2)-\left(\exp (x / 2) x^{\prime} \exp (-x / 2)\right. \\
& \quad=\exp (-x / 2)(\exp x)^{\prime} \exp (-x / 2) x-x \exp (-x / 2)(\exp x)^{\prime} \exp (-x / 2) .
\end{aligned}
$$

Wie wir oben bereits bemerkt haben, kommutieren $L_{x}$ und $R_{x}$, so dass gilt

$$
\left(\exp D_{x} / 2\right)=\left(\exp L_{x} / 2\right) \cdot \exp \left(-R_{x} / 2\right) .
$$

Damit lässt sich die Gleichung (4) schreiben als

$$
\left(\exp \left(D_{x} / 2\right)-\exp \left(-D_{x} / 2\right)\right) x^{\prime}=D_{x}\left(F_{x} x^{\prime}\right) .
$$


Wir nehmen jetzt die spezielle Kurve $x(t)=v+t w$ und evaluieren an der Stelle $t=0$. Dann ist $x^{\prime}(0)=w$, und es folgt für alle $v, w \in$ Mat

$$
D_{v}\left(F_{v} w\right)=D_{v}\left(\left(f\left(D_{v}\right) w\right) .\right.
$$

Dies war zu beweisen.

Theorem 3.4. Es sei $v \in \mathbf{S y m}$. Dann gilt $F_{v}=f\left(D_{v}\right)$ in $\mathbf{S y m}$, d.h. es besteht für alle $w \in \mathbf{S y m}$ die Identität

$$
\exp ^{\prime}(v) w=\exp (v / 2) \cdot f\left(D_{v}\right) w \cdot \exp (v / 2) .
$$

Beweis. Wir betrachten den Operator $h_{v}=F_{v}-f\left(D_{v}\right):$ Sym $\rightarrow$ Sym. Dieser ist symmetrisch bezüglich des tr-Skalarproduktes. Nach Lemma 3.3 ist $D_{v} h_{v}=0$. Damit ist das Bild unter $h_{v}$ enthalten in $E=\operatorname{ker} D_{v} \cap \mathbf{S y m}$. Da Sym endlich-dimensional ist, folgt Sym $=E \oplus E^{\perp}$, wo $E^{\perp}$ das orthogonale Komplement von $E$ in Sym bezeichnet. Da $h_{v}$ symmetrisch ist, bildet es $E^{\perp}$ nach $E^{\perp}$ ab. Aber $h_{v}$ bildet $E^{\perp}$ auch nach $E$ ab. Damit folgt $h_{v}=0$ auf $E^{\perp}$. Ferner besteht $E$ laut Definition aus allen Elementen $w \in \mathbf{S y m}$ mit $0=D_{v}(w)=v w-w v$. Damit folgt für $w \in E$ sofort $f\left(D_{v}\right) w=w=F_{v}(w)$, und es ist $h_{v}=0$ auf $E$. Die Abbildung $h_{v}$ ist also auf dem ganzen Raum Sym trivial. Dies war zu beweisen.

Theorem 3.5. Es sei $v \in \mathbf{S y m}$. Dann ist $D_{v}^{2}$ semi-positiv, und es gilt $f\left(D_{v}\right) \geq I$.

Beweis. Gemäss Lemma 3.2 gilt für $v \in \mathbf{S y m}$

$$
\left\langle D_{v}^{2} w, w\right\rangle_{\mathrm{tr}}=\operatorname{tr}\left(w v^{2} w-2 v w v w+v^{2} w^{2}\right)=2 \operatorname{tr}\left(v^{2} w^{2}-(v w)^{2}\right) .
$$

Die Semi-Positivität von $D_{v}^{2}$ folgt somit aus der Schwarzschen Eigenschaft von tr.

Die Potenzreihe $f(t)$ besitzt nur Glieder mit geraden Exponenten. Deshalb lässt sie sich schreiben als $f_{0}\left(t^{2}\right)$, wo ausserdem die Potenzreihe $f_{0}(t)$ positive Koeffizienten besitzt. Ferner ist $f_{0}(t) \geq 1$ für alle $t \geq 0$. Nach der Spektraleigenschaft folgt dann

$$
f\left(D_{v}\right)=f_{0}\left(D_{v}^{2}\right) \geq I .
$$

Dies war zu beweisen.

Theorem 3.6. Die Exponentialabbildung auf Sym ist semi-dilatierend, d.h. für alle $v, w \in \mathbf{S y m}$ und $p=\exp (v)$ gilt

$$
|w|_{\mathrm{tr}}^{2}=\operatorname{tr}\left(w^{2}\right) \leq \operatorname{tr}\left(\left(p^{-1} \exp ^{\prime}(v) w\right)^{2}\right)=\left|\exp ^{\prime}(v) w\right|_{\exp (v)} .
$$

Beweis. Für die rechte Seite der Gleichung erhalten wir mit Theorem 3.4:

$$
\begin{aligned}
\operatorname{tr}\left(\left(p^{-1} \exp ^{\prime}(v) w\right)^{2}\right) & =\operatorname{tr}\left(\exp (-v) \cdot \exp (v / 2) \cdot f\left(D_{v}\right) w \cdot \exp (v / 2)\right)^{2} \\
& =\operatorname{tr}\left(f\left(D_{v}\right) w\right)^{2} \\
& =\left|f\left(D_{v}\right) w\right|_{\text {tr }}^{2} .
\end{aligned}
$$

Die Anwendung von Theorem 3.5 vervollständigt dann den Beweis. 
Nach den Bemerkungen, die Theorem 2.1 folgen, ist damit auch der Beweis von Theorem 2.1 erbracht. Wir schliessen noch das folgende Korollar an.

Korollar 3.7. Für $v \in \mathbf{S y m}$ sind die Abbildungen

$$
F_{v} \quad \text { und } \exp ^{\prime}(v): \mathbf{S y m} \rightarrow \mathbf{S y m}
$$

lineare Automorphismen.

Beweis. Theorem 3.6 besagt, dass der Kern der Abbildung $\exp ^{\prime}(v)$ trivial ist; folglich ist $\exp ^{\prime}(v)$ ein Isomorphismus. Die Aussage über $F_{v}$ ergibt sich dann aus der Tatsache, dass diese Abbildung eine Zusammensetzung von $\exp ^{\prime}(v)$ mit invertierbaren Abbildungen ist. Damit ist der Beweis von Korollar 3.7 vollständig.

\section{Historische Anmerkungen}

Die Darstellung des obigen Materials folgt im wesentlichen einem Weg, der dem historischen entgegengesetzt ist. Es hat fast ein Jahrhundert gedauert, bis die hier behandelten Ideen ihre heutige Allgemeinheit und Einfachheit erreicht haben.

Historisch begannen die Dinge am Ende des 19. Jahrhunderts. Wie Klingenberg [K1 83/95] feststellt, hat Mangoldt [vM 1881] ein Resultat bewiesen, das heute das Cartan-Hadamard-Theorem für Flächen genannt wird. Mangoldts Veröffentlichung liegt also 15 Jahre vor derjenigen von Hadamard [Ha 1896], wo das Resultat noch einmal bewiesen wurde. Mangoldt verweist in seiner Arbeit sogar auf noch frühere Veröffentlichungen, Hadamard verweist auf Mangoldt, und Cartan, der in [Ca 28] allgemeine Riemannsche Mannigfaltigkeiten behandelt, verweist auf Mangoldt und Hadamard. Ich bin nicht fähig, die Originalarbeiten zu lesen.

Helgason [He 62, He 78] gibt Cartans Fixpunktsatz in der folgenden Form an, siehe [He 78], Chapter I, Theorem 13.5: Auf einer Riemannschen Mannigfaltigkeit mit seminegativer Krümmung besitzt eine kompakte Gruppe von Isometrien einen Fixpunkt. Helgason gibt dafür einen Beweis, der den Ideen von Cartan [Ca 28] folgt. Cartans unmittelbares Ziel war es, zu zeigen, dass alle maximalen kompakten Untergruppen einer halbeinfachen Liegruppe konjugiert sind. Mostow [Mo 53] gab eine ähnliche Darstellung, aber in einem spezielleren Rahmen. Alle diese Darstellungen machten in ihren Beweisen Gebrauch von einer Massenverteilung und deren Zentrum, wo wir in unserer Darstellung einfach vom Umkugelzentrum sprechen konnten. Dann formulierten Bruhat-Tits [BrT 72] ihren Fixpunktsatz, der hier als Theorem 1.2 erscheint, und machten in ihrem Beweis wesentlich vom Semi-Parallelogrammgesetz Gebrauch. Serre benützte schliesslich eine Variation dieses Beweises und die Aussage in unserem Theorem 1.1, um das jetzige allgemeine Resultat mit dem sehr einfachen Beweis zu erreichen. Ich weiss nicht, ob Serre dieses Resultat je veröffentlicht hat, aber Brown (siehe [Bro 89], Chapter VI, Theorem 2 von $\S 5$ ) schreibt es in genau der Formulierung, die wir hier gegeben haben, Serre zu.

Wir sehen also, wie eine Entwicklung von Ideen, die vor einem Jahrhundert begonnen hat, schliesslich zu einem sehr allgemeinen, aber elementaren Resultat über metrische Räume geführt hat. So konnte die Voraussetzung über die Kompaktheit abgeschwächt werden zu 
einer Voraussetzung über die Beschränktheit, und die komplizierte Bedingung über die Krümmung wurde durch das einfache Semi-Parallelogrammgesetz ersetzt. Ferner wurde das Zentrum der Massenverteilung, das in Cartans Darstellung und in vielen folgenden eine grosse Rolle spielt, nach Bruhat-Tits durch das Umkugelzentrum ersetzt.

Bruhat-Tits [BrT 72] haben einfach zusammenhängende Riemannsche Mannigfaltigkeiten mit semi-negativer Krümmung sogar durch das Semi-Parallelogrammgesetz charakterisieren können. Tatsächlich ist das lokale Semi-Parallelogrammgesetz äquivalent zu lokaler seminegativer Krümmung, und diese beiden Eigenschaften sind ihrerseits äquivalent zur Tatsache, dass die exp-Abbildung semi-dilatierend ist. Diese Erkenntnisse gaben in der Folge Anlass zur Entwicklung einer Krümmungstheorie in metrischen Räumen statt in Mannigfaltigkeiten. Für eine Darstellung dieser Theorie verweisen wir auf Ballmann [Ba 95]. Dort ist unser Theorem 1.1 ebenfalls zu finden; Ballmann verweist in diesem Zusammenhang auf Brown, siehe [Ba 95], Theorem 5.1 und Proposition 5.10 von Chapter I.

Die Entwicklung der Theorie symmetrischer Räume, für die $\mathbf{P o s}_{n}$ der Prototyp ist, verdankt man E. Cartan [Ca 28], [Ca 46], [Ca 51]. Mostow gab in [Mo 53] eine sehr elegante Darstellung eines Teils von Cartans Resultaten. In unserem Abschnitt 3 sind wir im wesentlichen der Darstellung von Mostow gefolgt.

Wir haben in den Abschnitten 2 und 3 im Raum der positiven Matrizen eine lokal variable Metrik eingeführt und haben damit gearbeitet. Der Leser kennt wahrscheinlich andere Beispiele ähnlicher Art, möglicherweise das folgende: Es sei $D$ die Einheitskreisscheibe in $\mathbf{R}^{2}$ und es bezeichne $r=r(x)$ für jeden Punkt $x \in D$ den euklidischen Abstand vom Mittelpunkt der Kreisscheibe. Dann definiert man die sogenannte Lobatschewsky- oder Poincaré-Metrik in $D$ durch

$$
|v|_{x}=\frac{1}{1-r^{2}}|v|_{\text {eukl }} \quad \text { mit } r=|x| .
$$

Es hängt also $|v|_{x}$ ab vom Abstand vom Ursprung, und es gilt $|v|_{x} \rightarrow \infty$ für $|x| \rightarrow 1$. Die Abhängigkeit von $x$ in der Einheitskreisscheibe ist hier von verhältnismässig einfacher Art, jedenfalls einfacher als die lokale Abhängigkeit der Spur-Metrik im Raum Pos ${ }_{n}$.

Das Skalarprodukt, das wir in $\mathbf{S y m}_{n}$ eingeführt haben, geht im wesentlichen auf Killing zurück. Die von diesem eingeführte Killing-Form ist aber auf kompliziertere Art definiert und unterscheidet sich vom tr-Skalarprodukt um einen konstanten Faktor. Am Ende des 19. Jahrhunderts, als die ersten Modelle der nichteuklidischen Geometrie entdeckt wurden, haben die Mathematiker solche lokal variablen Metriken eingeführt. In der Tat liefert die Einheitskreisscheibe $D$ mit der oben eingeführten Metrik ein Modell der nichteuklidischen Geometrie. Andere derartigen Metriken haben später u.a. in der Allgemeinen Relativitätstheorie eine grosse Rolle gespielt.

Acknowledgment. I am very grateful to U. Stammbach for the care he has given to the translation and the publication of my paper. 


\section{Literatur}

[Ba 95] W. Ballman, Lectures on Spaces of Nonpositive Curvature, Birkhäuser, 1995

[Bro 89] K. Brown, Buildings, Springer Verlag, 1989

[BrT 72] F. Bruhat and J. Tits, Groupes Réductifs sur un Corps Local I, Pub. IHES 41 (1972), pp. 5-251

[Ca 27a] E. Cartan, Sur une classe remarquable d'espaces de Riemann, Bull. Soc. Math. France 54 (1927) pp. $114-134$

[Ca 27b] E. Cartan, Sur certaines formes Riemanniennes remarquables des géometries à groupe fondamental simple, Ann. Sci. Ecole Norm. Sup. 44 (1927) pp. 345-467

[Ca 28/46] E. Cartan, Leçons sur la géométrie des espaces de Riemann, Gauthiers-Villars, Paris,1928; 2nd edition, 1946

[Ca 51] E. Cartan, Leçons sur la géométrie des espaces de Riemann II, Gauthiers-Villars, Paris, 1951

[Ha 86] J. Hadamard, Les surfaces à courbures opposées et leur lignes géodésiques, J. Math. Pures Appl. (5) 4 ( 1896) pp. 27-73

[He 62] S. Helgason, Differential geometry and symmetric spaces, Academic Press, 1962

[He 78] S. Helgason, Differential Geometry, Lie Groups, and Symmetric Spaces, Academic Press, 1978

[K1 83/95] W. Klingenberg, Riemannian Geometry, de Gruyter, 1983; second edition 1995

[vM 81] H. von Mangoldt, Über diejenigen Punkte auf positiv gekrümmten Flächen, welche die Eigenschaft haben, dass die von ihnen ausgehenden geodätischen Linien nie aufhören, kürzeste Linien zu sein, $J$. reine angew. Math. 91 (1881) pp. 23-52

[Mo 53] D. Mostow, Some new decomposition theorems for semisimple groups, Memoirs AMS, 1953

Serge Lang

Yale University

New Haven

Connecticut, 06520, USA 